Acta Cryst. (1954). 7, 505

\title{
The Lattice Spacings of Lead-rich Substitutional Solid Solutions
}

\author{
By C. Tyzack and G. V. Raynor \\ Department of Metallurgy, The University, Edgbaston, Birmingham 15, England
}

(Received 23 January 1954 and in revised form 18 March 1954)

\begin{abstract}
The lattice-spacing/composition curves for the lead-rich alloys of lead with antimony, bismuth, tin, indium, thallium, cadmium and mercury have been determined. Only bismuth expands the lead lattice. The distortions produced by equiatomic percentages of solute depend on the relative atomic volumes and relative valencies of the solute and solvent metals. Thus, for the solute metals of the second long period of the Periodic Table, the contractions produced by equal atomic percentages of solute decrease in the order cadmium, indium, tin, antimony; this is also the order in which the group valencies and the atomic volumes of the solute metals increase. Similarly, for mercury, thallium and bismuth, the contraction caused by mercury is greater than that caused by the same atomic percentage of thallium, while bismuth, the only solute for which the atomic volume is greater than that of lead, expands the lattice. The results are discussed with particular reference to the effect on lattice-spacing relationships of differences in atomic size and valency between the solute and solvent metals. No simple correlations are observed; it is concluded that lattice spacings in lead alloys are influenced by some factor connected with the electronic structures of the underlying ions of the component metals, in addition to factors already recognized in alloy systems based on solvent metals of lower valency and smaller atomic number.
\end{abstract}

\section{Introduction}

Investigations have already been carried out relating to the variation with composition of the lattice spacings of copper- and silver-rich alloys (Hume-Rothery, Lewin \& Reynolds, 1936; Owen \& Roberts, 1939), magnesium-rich alloys (Raynor, 1940, 1942; HumeRothery \& Raynor, 1940), and aluminium-rich alloys (Axon \& Hume-Rothery, 1948). Certain principles affecting the lattice spacings of alloys have become clear, although the phenomena encountered increase in complexity as the group valency of the solvent metal rises. Thus, lattice spacings in copper- and silverrich alloys depend mainly on the differences between the atomic diameters and the group valencies of the component metals, and interesting regularities are observed (Hume-Rothery et al., 1936; Raynor, 1949). For magnesium as solvent, effects due to overlap of electrons from the first Brillouin zone for the closepacked hexagonal structure predominate. In aluminium-rich solid solutions, more complicated effects associated with the relative sizes of the ions of the component metals, and the relative volumes available per valency electron in their respective structures, are observed. In spite of expected complexity, therefore, it is now of interest to examine lattice spacing relationships in a solvent of the fourth group of the Periodic Table. Attention has thus been directed to metallic lead as solvent, and the lattice spacings of the solid solutions in lead of antimony, bismuth, tin, indium, thallium, cadmium and mercury have been determined. The results are reported in the present paper.

\section{Experimental methods}

The materials used were as follows:

(i) Lead, of purity exceeding $99.995 \%$, kindly presented by the Cookson Lead and Antimony Co. Ltd.

(ii) Indium wire, spectroscopically pure, obtained from Messrs Johnson, Matthey and Co. Ltd.

(iii) Thallium wire, spectroscopically pure, obtained from Messrs Johnson, Matthey and Co. Ltd.

(iv) 'Chempur' Tin, $99.992 \%$ pure, obtained from Messrs Capper Pass and Son, Ltd.

(v) Mercury, spectroscopically pure, obtained from Messrs Johnson, Matthey and Co. Ltd.

(vi) Cadmium, spectroscopically pure, kindly presented by the Imperial Smelting Corporation, Avonmouth.

Alloys were prepared in $2 \mathrm{~g}$. quantities; the components were accurately weighed into thin-walled pyrex tubes provided with bulbs at one end sufficiently large to accommodate the unmolten metal. The tubes were evacuated, flushed with argon several times, and sealed in vacuo. They were then attached to a rotating shaft, inserted into the centre of a tubular resistance furnace whose temperature was accurately controlled, and rotated for $30 \mathrm{~min}$. at $10^{\circ} \mathrm{C}$. above the melting point of the higher-melting component. After cooling to some $5-10^{\circ} \mathrm{C}$. above the liquidus temperature for the alloy concerned, the molten metal was tipped into the cylindrical portion of the preparation tube, which was quenched into water. Cylindrical 
ingots with a fine-grained 'as-cast' microstructure were obtained. Since the pyrex tubes did not shatter on quenching, homogenizing heat treatments were carried out in the same tubes, and the alloys were finally quenched (or slowly cooled in the case of extensive solid solution formation). These annealed ingots, after extraction from the annealing tubes, differed in weight by less than $1 \mathrm{mg}$. from that of the component metals used, so that the intended compositions were accurately attained, as shown by subsequent chemical analysis.

Tests were carried out to ensure that a sufficient period was employed for homogenization. Small cylindrical specimens of typical alloys were annealed for successively longer periods until filings taken from the centre and the periphery gave lattice-spacing values identical within the limits of experimental error. The corresponding period was taken as the minimum permissible for the particular alloy system.

Filings of the alloys were prepared by conventional methods, and annealed in vacuo, at the same temperature as that from which the parent lump was quenched, to relieve introduced stresses. Specimens were exposed to copper, cobalt, or iron $K \alpha$ radiation in 9 or $19 \mathrm{~cm}$. diameter cylindrical cameras, accurately standardized by direct measurement and also against the lattice spacing of pure silver. The camera temperature was kept constant throughout exposures, and the mean lattice spacings derived were corrected to a common temperature of $20^{\circ} \mathrm{C}$. Systematic errors were minimized by the use of the Nelson-Riley (1945) extrapolation function, and the spacings are estimated to be accurate to within $\pm 0 \cdot 0001 \mathrm{kX}$.

The values of the wavelengths and other physical constants employed in the lattice-spacing work were:

$\begin{array}{lll} & K \alpha_{1}(\mathrm{kX} .) & K \alpha_{2}(\mathrm{kX} .) \\ \mathrm{Cu}: & 1.537395 & 1.541232 \\ \mathrm{Co}: & 1.78529 & 1.78919 \\ \mathrm{Fe}: & 1.932076 & 1.936012\end{array}$

Linear thermal expansion coefficient of silver:

$18.8 \times 10^{-6}$ per deg. C.

Linear thermal expansion coefficient of lead:

$29 \times 10^{-6}$ per deg. C.

Representative specimens from each alloy system were analysed chemically by Messrs Johnson, Matthey and Co. Ltd. Since the analytical results agreed with the synthetic compositions to within the limits of accuracy of the analytical methods, and since the totals of the solute and solvent percentages were satisfactory, it has been considered justifiable to use synthetic compositions, which are estimated to be correct to $\pm 0 \cdot 10$ atomic per cent unless otherwise stated, in plotting the results.

\section{Experimental results}

\section{(a) The system lead-bismuth}

Alloys were cast at intervals of 1 atomic \% from 0 to 40 atomic \% of bismuth. The filings were annealed for several hours at the lump annealing temperature, and quenched into ice water. Six alloys with compositions in the range $21 \cdot 88-26 \cdot 84$ atomic \% of bismuth were micrographically duplex after annealing for 19 days at $115-117^{\circ} \mathrm{C}$; t the diffraction patterns showed the presence of a close-packed hexagonal structure, which was obtained pure at 28.83 and 30.78 atomic \% of bismuth. The lattice spacings of the former alloy were: $a=3.5013 \mathrm{kX} ., c=5.7054 \mathrm{kX}$., $c / a=1.6295$ at $20^{\circ} \mathrm{C}$. Three alloys exceeding a bismuth content of 34.81 atomic \% contained the close-packed hexagonal phase and the bismuth-rich solid solution. These results are consistent with the equilibrium diagram proposed by van Hofe \& Hanemann (1940). The lattice spacings determined in the present work (Fig. 1) are

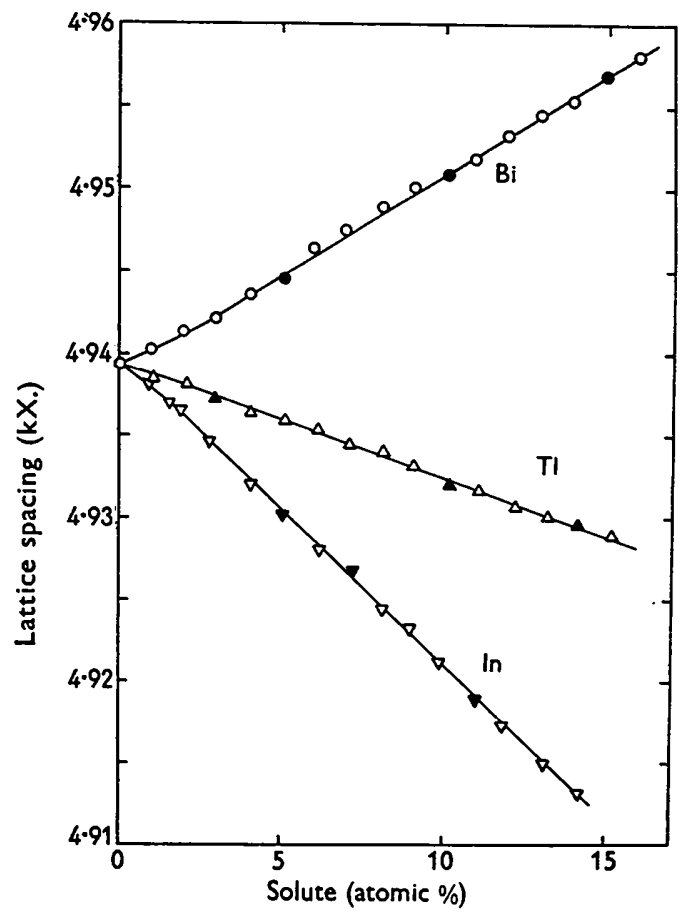

Fig. 1. Lattice spacings of lead-rich lead-bismuth, leadthallium, and lead-indium alloys. Analyzed alloys are distinguished by full symbols.

in qualitative agreement with the less detailed work of van Hofe \& Hanemann, and with the results published by Hayasi (1939). Within the limits of experimental error, the variation of lattice spacing with compositions is linear.

\section{(b) The system lead-thallium}

The solid solubility of thallium in lead is very extensive; Tang \& Pauling (1952) show that the facecentred cubic solid solution orders at the compositions $\mathrm{PbTl}_{7}$ and $\mathrm{PbTl}_{3}$, and persists up to 88 atomic $\%$ of thallium at room temperature. In the present work, the composition range $0-15$ atomic $\%$ of thallium was examined. Special care was taken in the evacuation of casting and annealing tubes, in view of the oxidizable 
nature of thallium, and all filing operations were carried out under carbon tetrachloride. The latticespacing measurements are plotted in Fig. 1.

\section{(c) The system lead-indium}

According to Valentiner \& Haberstroh (1938), the face-centred cubic solid solution of indium in lead exceeds 64.5 atomic $\%$ of indium, but an indium-rich phase is present at 69 atomic \%. In the present work, alloys were prepared in the range 0-14.2 atomic \% of indium; all were annealed for 3 months at $150^{\circ} \mathrm{C}$. and quenched in ice water. The lattice-spacing/composition curve (Fig. 1) again appears to be linear.

In this system, analytical and synthetic compositions of selected alloys agreed less well than for other systems. In view of the satisfactory consistency of the lattice-spacing results, however, and the very slight changes in weight of the metals on alloying and annealing, the points plotted in Fig. 1 are regarded as accurate to within $\pm 0 \cdot 15$ atomic $\%$.

\section{(d) The system lead-mercury}

Little reliable information exists about this system. Solidus-curve determinations by Puschin (1903), Jänecke (1907) and Babinski (1906) suggest that the mercury content of the lead-rich solid solution in equilibrium with the liquid reaches a maximum of approximately 35 atomic $\%$ at the peritectic temperature of $-37 \cdot 6^{\circ} \mathrm{C}$. Analysis of solid material extracted from mercury-rich liquid alloys (Fay \& North, 1901) suggests the presence of an intermediate phase of uncertain composition, but this evidence has been disputed. The present experiments embraced alloys containing 0-33 atomic \% of mercury. All were annealed for 3 weeks at $93-95^{\circ} \mathrm{C}$., and quenched in ice water; no chilled liquid was micrographically visible after quenching. The lattice-spacing/composition curve is a straight line, from which no experimental point varies by more than $0.0004 \mathrm{kX}$., over the whole range up to $16 \cdot 5$ atomic $\%$ of mercury, and can be represented by the equation

$$
a_{\mathrm{Pb}-\mathrm{Hg}}=4.9393-0.00356(\mathrm{Hg}),
$$

where $(\mathrm{Hg})$ is the atomic percentage of mercury, and $a_{\mathrm{Pb}-\mathrm{Hg}}$ is in $\mathrm{kX}$. For this reason, a diagram is considered unnecessary.

Two further alloys were investigated, with the same heat treatment as before. That containing 25.85 atomic $\%$ of mercury was metallographically duplex, giving a double diffraction pattern which was indexed in terms of the lead-rich structure $(a=4.8551 \mathrm{kX}$. at $20^{\circ} \mathrm{C}$.) and a face-centred tetragonal structure. The alloy containing 33.87 atomic $\%$ of mercury was homogeneous, large-grained and brittle, and gave a single diffraction pattern, corresponding to a facecentred tetragonal structure with the following unitcell dimensions $\left(\mathrm{kX}\right.$. at $\left.20^{\circ}\right)$ :

$$
a=4.9715, c=4.5027 \mathrm{kX} \text {; } c / a=0.9057 \text {. }
$$

An intermediate phase thus exists at 33 atomic $\%$ of mercury, and may be analogous to the face-centred cubic $\mathrm{NaPb}_{3}$, which is stable over the range 27-33 atomic \% of sodium in the system lead-sodium. Since the new phase is stable at $93-95^{\circ} \mathrm{C}$., it must be formed peritectically from the liquid and the lead-rich solid solution at some higher temperature. If the linearity of the lattice spacings of the lead-rich solution with composition continues up to the solubility limit of mercury in lead, the approximate solid solubility is 24 atomic \% of mercury, at $93-95^{\circ} \mathrm{C}$.

\section{(e) The system lead-antimony}

In this system the solid solution is limited, reaching a maximum of $5 \cdot 8$ atomic $\%$ of antimony at $247^{\circ} \mathrm{C}$. (the eutectic temperature), and falling to approximately 3 atomic \% at room temperature. Only three alloys, annealed for 3 months at $210^{\circ} \mathrm{C}$. and quenched in ice water, were investigated. All were micrographically homogeneous, and the lattice-spacing/composition curve is represented by the equation

$$
a_{\mathrm{Pb}-\mathrm{Sb}}=4 \cdot 9393-0 \cdot 00054(\mathrm{Sb}) ;
$$

$(\mathrm{Sb})$ is the atomic percentage of antimony, and $a_{\mathrm{Pb}-\mathrm{Sn}}$ is in $\mathrm{kX}$.

\section{(f) The system lead-tin}

The maximum solid solubility of tin in lead is $29 \cdot 3$ atomic $\%$ at the eutectic temperature of $183^{\circ} \mathrm{C}$. (Borelius, Larris \& Ohlsson, 1944). The solubility decreases rapidly with falling temperature, and does not exceed 3 atomic \% at room temperature. Alloys were therefore prepared, annealed for 3 months at $210^{\circ} \mathrm{C}$., and quenched in ice water. It was impossible to quench alloys containing more than 5 atomic \% of tin without partial break-down of the solid solution, and accurate lattice spacings could not be obtained. Below this composition rapid quenching preserved the supersaturated solid solution, and no evidence was found of precipitation at room temperature within a period of two days after quenching. Diffraction patterns were obtained as soon as possible after quenching; owing, however, to the possibility of decomposition on quenching, duplicate or triplicate experiments were carricd out using different samples of quenched filings for each alloy. Some slight variability in the lattice spacing was observed; thus three samples of an alloy containing 4.90 atomic \% of tin gave lattice spacings of $4.9322,4.9326$ and $4.9320 \mathrm{kX}$. The mean curve drawn through the experimental points shows no significant departure from a straight line

$$
\left(a_{\mathrm{Pb}-\mathrm{Sn}}=4.9393-0.00139(\mathrm{Sn}),\right.
$$

where the units are the same as previously), and is in good agreement with the results of Obinata \& Schmid (1933). 


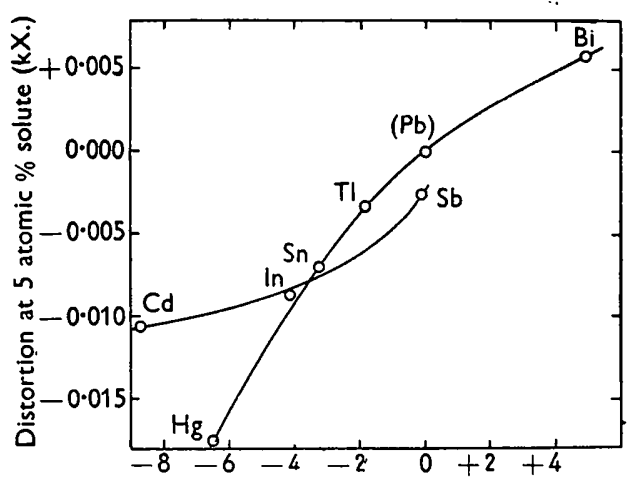

(Atomic volume of solute)-(Atomic volume of lead)

$\left(k X .{ }^{3}\right)$

(o)

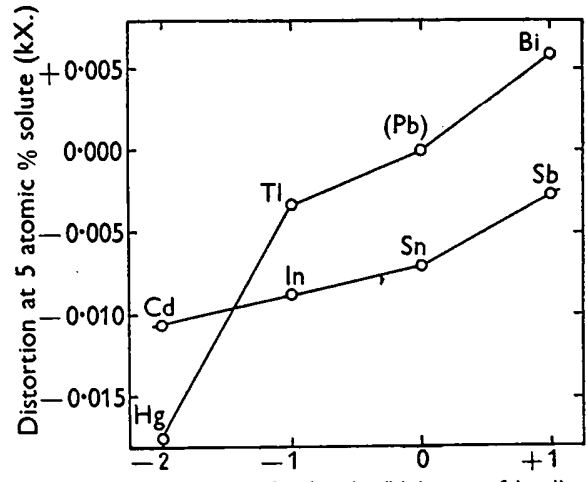

(Valency of solute)-(Valency of lead)

(b)

Fig. 2. Relative distortions of lead lattice by 5 atomic \% of solute, plotted against (a) (Atomic volume of solute)-(Atomic volume of lead), (b) (Group valency of solute)-(Group valency of lead).

\section{(g) The system lead-cadmium}

The solid solubility of cadmium in lead is approximately 5.9 atomic \% at the eutectic temperature of $248^{\circ} \mathrm{C}$., but falls to about 1 atomic \% at room temperature. Alloys containing up to 5 atomic \% of cadmium were examined, after annealing for 2 months at $210^{\circ} \mathrm{C}$. and quenching. Those with more than $1 \cdot 2$ atomic $\%$ of cadmium gave diffraction patterns containing lines due to a second phase in addition to the lead-rich solid solution. Similar effects occurred in a further series of experiments, and accurate spacings were obtained only for two alloys. The specimens of synthetic composition $1 \cdot 14$ and 1.28 atomic \% of cadmium gave lattice spacings of 4.9365 and 4.9367 $\mathrm{kX}$. at $20^{\circ} \mathrm{C}$. respectively; the analysed composition of the latter alloy was $\mathbf{1 \cdot 2 5}$ atomic $\%$ of cadmium.

\section{Discussion}

Of the solute elements examined in the present work, bismuth expands the face-centred cubic lattice of lead, and the remainder contract it. The lattice-spacing/composition curves are linear within the limits of experimental error.

To compare the lattice distortions caused by the solutes studied, it is convenient to consider a standard composition of 5 atomic \% of solute; this involves short extrapolations of the curves determined for the lead-antimony and lead-cadmium systems. In Fig. 2(a) the distortions of the lead lattice by 5 atomic $\%$ of solute are plotted against the difference between the atomic volumes of solute and solvent;* Fig. 2(b) shows the same distortions plotted against the difference in group valency between solute and solvent. For solutes belonging to a given period of the Periodic Table, the distortions are related to the relative atomic volumes and valencies of the solvent and solute metals. Thus, for mercury, thallium and bismuth, a positive distor-

\footnotetext{
* The atomic volume is defined as the volume of the unit cell, divided by the number of atoms per unit cell.
}

tion is observed only where the atomic volume of the solute is larger than that of the solvent; the negative distortions caused by mercury and thallium are approximately proportional to the difference between the atomic volumes of the solute metals and that of the solvent. For cadmium, indium, tin and antimony, the contractions caused by equiatomic percentages are in the order of the differences in atomic volume between solute and solvent. In both series, the contraction of the lattice is more marked as the valency of the solute metal decreases.

It is of interest to examine whether the effects of relative atomic sizes and of relative valencies may be separately discussed. In earlier work on copper, silver and gold solid solutions (Raynor, 1949), it was assumed that, in the absence of any effects due to the valency difference between the components, the mean closest distance of approach in an alloy containing an atom fraction $n$ of solute metal $B$ in a solvent $A$ could be approximated by the expression:

$$
d_{\text {mean }}^{\prime}=n d_{B}+(1-n) d_{A},
$$

where the $d$ 's represent closest distances of approach of atoms in the respective crystal structures. The difference

$$
\Delta d=d_{\text {mean }}(\text { obs. })-d_{\text {mean }}^{\prime}
$$

may then be taken as a measure of the effect of the solute valency. In such an analysis difficulties arise in assessing the correct size of the solute atom to be employed in calculating the distortion to be expected from atomic-size effects only, especially when the coordination numbers of the solute and solvent metals differ, or when the crystal structure of the solute metal is complex and gives rise to more than one distance of approach of atoms. Three approaches to the problem may be examined.

(i) The closest distances of approach of atoms in the crystal structures of the elements may be assumed in calculating the distortion expected from relative 
atomic-size effects. Then, taking the following closest distances of approach:

\begin{tabular}{|c|c|c|c|}
\hline $\mathrm{Cd}$ & $2.973 \mathrm{kX}$ & $\mathrm{Hg}$ & $3.009 \mathrm{kX}$. \\
\hline In & $3 \cdot 24$ & $\mathrm{Tl}$ & $3 \cdot 401$ \\
\hline Sn & $3 \cdot 016$ & - & 一 \\
\hline & $2 \cdot 898$ & $\mathrm{Bi}$ & $3 \cdot 105$ \\
\hline
\end{tabular}

$\Delta a\left(=a_{\text {obs. }}-a_{\text {calc. }}\right)$ for a constant solute percentage of 5 atomic \% may be plotted against the group valency for each metal, as in Fig. 3 (full lines). In their respective periods the three-valent solutes indium and thallium correspond to the smallest $\Delta a$ values; the five-valent elements antimony and bismuth have the largest $\Delta a$ values. These $\Delta a$ values are, however, in all cases positive, in marked contrast to the behaviour of magnesium- and aluminium-rich alloys, where $\Delta a$ is generally positive where the group valency of the solute exceeds that of the solvent, and negative where the reverse is the case.

It is often assumed that metallic indium, thallium and white tin are incompletely ionized, and that the interatomic distances corresponding to the completely ionized states are approximately 0.2 to $0.3 \mathrm{kX}$. smaller. It is most uncertain whether this correction should be made in the present case, but if it were made, $\Delta a$ in Fig. 3 for indium would be intermediate between the $\Delta a$ values for cadmium and antimony, while $\Delta a$ for thallium would be intermediate between the values for mercury and bismuth. The $\Delta a$ value obtained by assuming complete ionization for tin, however, is very large, and exceeds that of antimony.

Fig. 3 (full lines) is abnormal in that the $\Delta a$ value

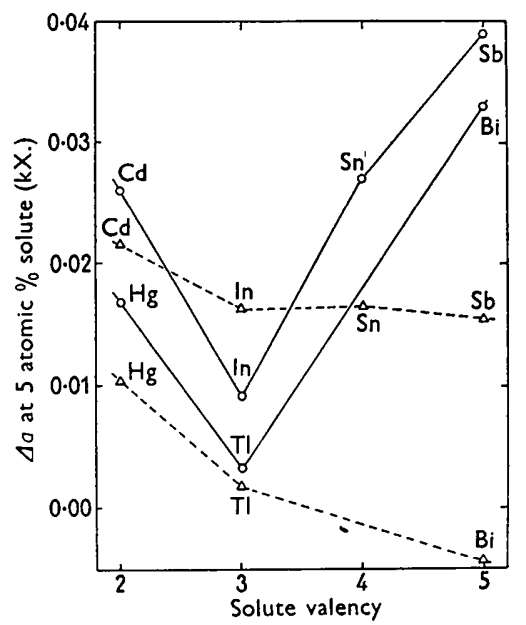

Fig. 3. $\Delta a$ at 5 atomic $\%$ of solute plotted against solute valency, assuming group valencies. Full lines: assuming closest distances of approach in calculating $\Delta \alpha$; broken lines: assuming Goldschmidt atomic diameters.

for tin, which has the same group valency as lead, is large, whereas mixtures of metals of equal valency in general give very small $\Delta a$ values. It is possible that in lead-rich alloys lead and tin are effectively two-valent, and indium and thallium effectively univalent, owing to the stability of the $5 s$ and $6 s$ subgroups of the electronic distribution. Assuming these valencies, $\Delta a$ varies with solute valency as in Fig. $4 ; \Delta a$ increases as the

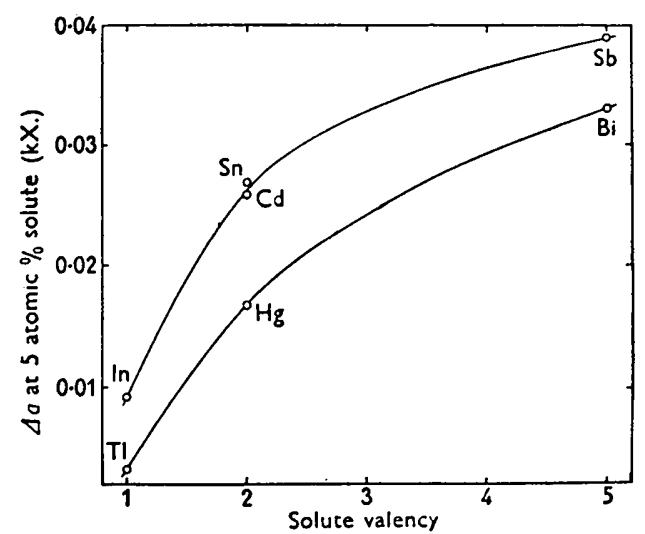

Fig. 4. $\Delta a$ at 5 atomic $\%$ of solute plotted against solute valency, assuming effective valencies of 2 for lead and tin, and 1 for indium and thallium.

valency increases, but the large $\Delta a$ for solutes assumed to be of the same effective valency as lead, and positive $\Delta a$ values for indium and thallium, are still abnormal.

(ii) Since the structure of lead corresponds to coordination number 12, the expected effect of atomicsize differences on the lead-rich lattice spacings should possibly be calculated assuming Goldschmidt atomic diameters, which also correspond with coordination number 12. The $\Delta a$ values at 5 atomic $\%$ of solute obtained in this way are also shown in Fig. 3 (broken lines). The deviations are again positive, except for bismuth.

(iii) Fig. 2 suggests a correlation between the distortions produced by equal atomic percentages of solute and the atomic volume of the solute. We may make a further assumption, therefore, that the effect of differences in the atomic sizes of the solute and solvent metals on the lattice spacings of lead-rich alloys is approximately given by an additive relationship between the atomic volumes of the components. The difference $\Delta V$ between the observed and calculated

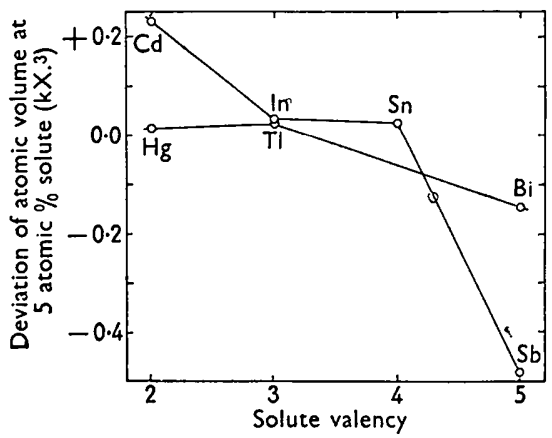

Fig. 5. Deviations of atomic volumes of alloys at 5 atomic $\%$ of solute from straight line joining atomic volumes of components, plotted against solute valency (assuming group valencies). 
Table 1

$\begin{array}{ccc}\text { Solute } & V_{e}\left(\mathrm{kX} .{ }^{3}\right) & \text { Effect of ionic radius } \\ \mathrm{Bi} & 7 \cdot 034 & \text { Contraction } \\ \mathrm{Sb} & 6 \cdot 006 & \text { Contraction } \\ \mathrm{Sn} & 6 \cdot 72 & \text { Contraction } \\ \mathrm{Tl} & 9 \cdot 47 & \text { Expansion } \\ \mathrm{In} & 8 \cdot 663 & \text { Little effect } \\ \mathrm{Hg} & 11 \cdot 73 & \text { Expansion } \\ \mathrm{Cd} & 10 \cdot 73 & \text { Expansion }\end{array}$

Apparent atomic
diameter $(\mathrm{kX}$.
$\mathbf{3} \cdot 577$
$\mathbf{3} \cdot 461$
$\mathbf{3} 400$
$\mathbf{3} \cdot 442$
$\mathbf{3 \cdot 3 6 4}$
$\mathbf{3 \cdot 2 4 8}$
$\mathbf{3 \cdot 3 3 5}$

Goldschmidt atomic diameter in solute (kX.) $3 \cdot 64$ 3.228 $3 \cdot 164$ $3 \cdot 42$ $3 \cdot 14$ $3 \cdot 10$ $3 \cdot 042$ atomic volumes at 5 atomic \% of solute is plotted against solute valency in Fig. 5. The interesting feature is that the deviations of the atomic volumes of the alloys from a straight line joining the atomic volumes of the components are very small, positive, and of similar magnitude for the systems lead-mercury, leadindium, lead-thallium and lead-tin. Large negative deviations are shown for lead-bismuth and leadantimony alloys, and a large positive deviation for the system lead-cadmium.

Attempted analysis of the separate influences of relative atomic size effects and relative valency effects, therefore, leads to no clear correlations, whether the size effects are assessed in terms of closest distances of approach of atoms, Goldschmidt atomic diameters, or atomic volumes. Fig. 2 suggests strongly, however, that both size and valency factors are involved.

For aluminium-rich alloys, Axon \& Hume-Rothery (1948) suggest that, if the volume per valency electron $\left(V_{e}\right)$ for the solute metal exceeds that for the solvent metal, the apparent atomic diameter of the solute metal, obtained by extrapolating the solvent-rich lattice-spacing/composition curve to $100 \%$ of solute, is less than the atomic diameter in the pure solute; the reverse tendency is also observed. At the same time, if the ionic radius of the solute is larger than that of the solvent, the effect is to increase the apparent atomic diameter of the former, and conversely. Leadrich solid solutions have been examined from this point of view. The relevant data are given in Table 1 , assuming the group valencies for the metals tabulated, and a $V_{e}$ value of $7.535 \mathrm{kX} .^{3}$ per electron for lead.

Bearing in mind that the effect of ionic radius is likely to be marked only when a relatively large ion is being forced into a structure in which its electron cloud begins to overlap the electron cloud of the ions of the solvent, the apparent atomic diameter values for thallium, indium, mercury and cadmium would be expected to be less than the atomic diameters in the crystals of the elements, whereas for all solutes listed in Table 1, the apparent atomic diameter values exceed the closest distances of approach of atoms in the solute metals, and also, except for bismuth, exceed the Goldschmidt diameter corresponding to coordination number 12.

The lattice spacings of lead-rich alloys are therefore difficult to interpret on the basis of previous treatments of lattice-spacing relationships. In particular, the abnormally large value of the apparent atomic diameter for tin, which has the same group valency as lead, requires explanation. The effects observed for solute metals from the second long period of the Periodic Table differ from those observed for third long period metals, and this suggests that some factor connected with the structures of the underlying ions may be responsible for the lack of regular behaviour.

The work reported forms part of a general programme in progress in the authors' laboratory, and grateful acknowledgement for generous financial assistance and other facilities which have aided the research is made to the Royal Society, the Chemical Society, Imperial Chemical Industries, Ltd, and the Department of Scientific and Industrial Research.

\section{References}

Axon, H. J. \& Hume-Rothery, W. (1948). Proc. Roy. Soc. A, 193, 1.

Babinski, J. J. (1906). Dissertation, Leipzig.

Borelius, G., Larris, F. \& OHLsson, E. (1944). Ark. Mat. Astr. Fys. 31 A, No. 10.

FAy, H. \& North, E. (1901). Amer. Chem. J. 25, 216. Hayasi, M. (1939). Nippon Kink. Gakk. 3, 123.

Hofe, H. van \& Hanemand, H. (1940). Z. Metallk. 32 , 112.

HUMe-Rothery, W., Lewin, G. F. \& Reynolds, P. W. (1936). Proc. Roy. Soc. A, 157, 167.

Hume-Rothery, W. \& Raynor, G. V. (1940). Proc. Roy. Soc. A, 177, 27.

JäNECKE, E. (1907). Z. phys. Chem. 60, 400.

Nelson, J. B. \& Rmey, D. P. (1945). Proc. Roy. Soc. $A, 57,160$.

Obinata, I. \& Schmid, E. (1933). Metallwirtschaft, 12, 101.

Owen, E. A. \& Roberts, E. W. (1939). Phil. Mag. (7), 27, 294.

Puschin, N. A. (1903). Z. anorg. Chem. 36, 201.

Raynor, G. V. (1940). Proc. Roy. Soc. A, 174, 457.

Raynor, G. V. (1942). Proc. Roy. Soc. A, 180, 107.

Raynor, G. V. (1949). Trans. Faraday Soc. 45, 698.

Tang, Y.-C. \& Pauling, L. (1952). Acta Cryst. 5, 39.

Valentiner, S. \& Haberstroh, A. (1938). Z. Phys. 110, 727. 